\title{
1 Sex Chromosome Heteromorphism and the Fast-X Effect in Poeciliids
}

2 Iulia Darolti ${ }^{1}$, Lydia J. M. Fong ${ }^{1}$, Judith E. Mank ${ }^{1,2}$

31 Department of Zoology and Biodiversity Research Centre, University of British Columbia,

4 Vancouver, Canada

52 Centre for Ecology and Conservation, College of Life and Environmental Sciences,

6 University of Exeter, Cornwall, United Kingdom

7 Corresponding author: Iulia Darolti, e-mail: darolti@zoology.ubc.ca 


\section{Abstract}

9 An accelerated rate of sequence evolution on the $X$ chromosome compared to autosomes,

10 known as Fast-X evolution, has been observed in a range of heteromorphic sex chromosomes.

11 However, it remains unclear how early in the process of sex chromosome differentiation the

12 Fast-X effect becomes detectible. Recently, we uncovered an extreme variation in sex

13 chromosome heteromorphism across Poeciliid fish species. The common guppy, Poecilia

14 reticulata, Endler's guppy, $P$. wingei, and the swamp guppy, $P$. picta, appear to share the same

$15 \mathrm{XY}$ system and exhibit a remarkable range of heteromorphism. The sex chromosome system

16 is absent in recent outgroups, including P. latipinna and Gambusia holbrooki. We combined

17 analyses of sequence divergence and polymorphism data across Poeciliids to investigate $\mathrm{X}$

18 chromosome evolution as a function of hemizygosity and reveal the causes for Fast-X effects.

19 Consistent with the extent of $Y$ degeneration in each species, we detect higher rates of

20 divergence on the $\mathrm{X}$ relative to autosomes and a strong Fast- $\mathrm{X}$ effect in $P$. picta, while no

21 change in the rate of evolution of X-linked relative to autosomal genes in P. reticulata. In $P$.

22 wingei, the species with intermediate sex chromosome differentiation, we see an increase in

23 the rate of nonsynonymous substitutions on the older stratum of divergence only. We also

24 use our comparative approach to test different models for the origin of the sex chromosomes

25 in this clade. Taken together, our study reveals an important role of hemizygosity in Fast-X and suggests a single, recent origin of the sex chromosome system in this clade.

27 Keywords: sex chromosome evolution, heteromorphy, hemizygosity 
Introduction

Owing to their unusual inheritance pattern and hemizygosity in males, $\mathrm{X}$ chromosomes display many distinct evolutionary properties compared to the rest of the genome (Charlesworth et al. 1987; Vicoso and Charlesworth 2006). The strength of selection and genetic drift, as well as the role of dominance and effective population size, are expected to differ between sex chromosomes and autosomes (Kirkpatrick and Hall 2004; Mank et al. 2010; Meisel and Connallon 2013). Comparing differences in the evolution of sex-linked and autosomal loci is important for understanding patterns of mutation and selection acting across the genome.

An elevated rate of coding sequence evolution on the $X$ chromosome relative to the autosomes, referred to as the Fast-X effect (or Fast-Z in the case of ZW systems) has been observed in a diversity of organisms, including humans (Lu and Wu 2005), primates (Stevenson et al. 2007), mice (Kousathanas et al. 2014), birds (Mank et al. 2007; Mank et al. 2010; Wright et al. 2015), Drosophila (Mank et al. 2010; Ávila et al. 2014; Charlesworth et al. 2018), aphids (Jaquiéry et al. 2018), spiders (Bechsgaard et al. 2019), and lepidoptera (Pinharanda et al., 2019; Sackton et al., 2014). However, the magnitude of this effect can vary substantially across species due to demographic factors, differences in mating system or regulatory mechanisms acting on the sex chromosomes (Mank et al. 2010). Notably, all the cases of Fast-X above were observed in highly heteromorphic sex chromosomes, where very few genes, if any, remain on the $\mathrm{Y}$ chromosome and the $\mathrm{X}$ is largely hemizygous in the heterogametic sex.

There are two potential causes of Fast- $X$. The single functional copy of the $X$ chromosome in males leads to hemizygous exposure of genes and therefore stronger purifying selection against recessive deleterious mutations and positive selection for recessive beneficial ones expressed in males (Charlesworth et al. 1987). This adaptive cause of Fast-X is mainly expected in species with heteromorphic sex chromosomes and highly degenerated $\mathrm{Y}$ chromosomes, as a large proportion of X-linked genes will be hemizygous in males. Alternatively, in every male and female pair, there are only three $\mathrm{X}$ chromosomes to four copies of each autosome, and this varies substantially based on mating system and type of heterogamety (Vicoso and Charlesworth 2009; Mank et al. 2010; Wright and Mank 2013; Wright et al. 2015). This reduced effective population size of the $X$ relative to the autosomes diminishes the relative power of 
deleterious mutations on the $X$ chromosome (Charlesworth et al. 1987). This non-adaptive cause of Fast-X does not necessarily require male hemizygosity, just recombination suppression between the $\mathrm{X}$ and $\mathrm{Y}$ chromosomes and differences in effective population size between $\mathrm{X}$-linked and autosomal loci, and could apply to $\mathrm{X}$ loci where the $\mathrm{Y}$ copy is expressed and remains functional.

Recently, we uncovered an extreme variation across Poeciliids in the rate of sex chromosome degeneration and dosage compensation. The same chromosome pair acts as the XY system in the common guppy, Poecilia reticulata, its sister species Endler's guppy, $P$. wingei, and the more distantly related swamp guppy, $P$. picta, all of which last shared a common ancestor roughly 20 million years ago (Darolti et al. 2019). Notably, P. latipinna and G. holbrooki, recent outgroup species to this clade, do not show evidence of a sex chromosome system on the same chromosome (Darolti et al. 2019), and therefore we hypothesized based on parsimony that the sex chromosome system arose roughly 20 mya (supplementary Fig. S1, Parsimony Model). Since this origin, the sex chromosomes of $P$. reticulata and $P$. wingei have remained largely homomorphic, with little sequence differentiation between the $\mathrm{X}$ and the $\mathrm{Y}$. In contrast, the $P$. picta sex chromosomes are completely nonrecombining (with the exception of a small pseudoautosomal region at the distal end, $>20 \mathrm{Mb}$ ) and the $\mathrm{Y}$ chromosome has undergone substantial degeneration, leaving most of the $X$ chromosome in males effectively hemizygous (Darolti et al. 2019). Moreover, P. picta exhibits complete dosage compensation, a pattern that has also been observed in its sister taxon, P. parae (Metzger et al. 2021; Sandkam et al. 2021), which is predicted to accentuate the Fast-X effect (Charlesworth et al. 1987; Mank et al. 2010). The range of sex chromosome differentiation present in this clade allows us to investigate patterns of $X$ chromosome coding sequence evolution and to test causes underlying Fast-X effects.

In contrast to the Parsimony Model, it has recently been proposed that the sex chromosomes of $P$. reticulata represent a recent turnover event, and that the highly diverged $P$. picta and $P$. parae system is in fact ancient (Charlesworth et al. 2021). This Turnover Model (supplementary Fig. S1) posits that the P. picta $\mathrm{X}$ chromosome arose well before the immediate ancestor of $P$. parae, $P$. picta, $P$. reticulata and $P$. wingei, and slowly diverged over time resulting in the heteromorphism observed. This model supposes that the homomorphism observed in $P$. reticulata and $P$. wingei represents a turnover event, where 
the current guppy $X$ and $Y$ chromosomes arose from the ancestral $X$ of $P$. picta. Sex chromosome turnovers are frequent in many animal groups (Bachtrog et al. 2014), so the model is plausible.

However, the unique molecular and evolutionary signatures that accumulate on sex chromosomes remain observable even when they revert to being autosomes (Vicoso and Bachtrog 2013). That means that if the Turnover Model is true, Fast-X patterns, evolving over long periods of time in the distant ancestor of $P$. picta, would also remain detectible on the $\mathrm{X}$ chromosome in P. reticulata and $P$. wingei after turnover. Furthermore, the Turnover Model requires additional turnover events in several other taxa within the clade that do not share the same sex chromosome as P. picta (supplementary Fig. S1), and we would also expect these species to exhibit ancient patterns of Fast-X on the chromosome, which is fully autosomal. Our comparative dataset allows us to also test these alternative hypotheses for the origin of the guppy sex chromosomes.

Using a combination of sequence divergence, polymorphism and expression data analyses across Poeciliid species, we estimate rates of gene sequence evolution across the genome and assess the presence of Fast-X evolution in each system. Consistent with the extent of $Y$ degeneration, we find significantly higher rates of $X$ coding sequence evolution compared to the autosomes and a strong signal of Fast-X effect in $P$. picta, potentially accelerated by the evolution of chromosome wide dosage compensation in this system. The absence of a consistent Fast-X evolution in $P$. reticulata and $P$. wingei, as well as in the outgroup species $P$. latipinna and G. holbrooki, argues against the Turnover Model.

\section{Results}

\section{Strong signal of Fast-X evolution in the heteromorphic sex chromosomes of $\boldsymbol{P}$. picta}

112 We first assessed the strength of Fast-X in our study species with heteromorphic sex

113 chromosomes. The extensive $\mathrm{X}$ chromosome hemizygosity of $P$. picta males is expected to 114 accentuate the strength of Fast-X evolution in this species (Vicoso and Charlesworth 2006), as

115 is the mechanism of complete $X$ chromosome dosage compensation (Mank et al. 2010). 116 Indeed, our analysis in $P$. picta revealed a greater mutation rate on the $\mathrm{X}$ chromosome, 117 excluding genes in the pseudoautosomal region (PAR) (see methods), compared to the 118 autosomes, as shown through the significantly higher rate of nonsynonymous substitutions 
119 for X-linked loci (1,000 replicates permutation test, $p<0.001$; supplementary Table S1). $P$.

120 picta also exhibits a significantly elevated rate of divergence $\left(d_{N} / d_{S}\right)$ on the $\mathrm{X}$ chromosome 121 relative to the rest of the genome (Fig. 1C) and a strong Fast-X effect (Fig. 2), calculated as the ratio of the rate of nonsynonymous over synonymous substitutions for the $\mathrm{X}$ chromosome relative to that for the autosomes.

\section{Absence of Fast-X effect in species with homomorphic sex chromosomes}

We next asked whether patterns of coding sequence evolution are different between $P$. picta and the species with homomorphic sex chromosomes, $P$. reticulata and $P$. wingei. Previous work has indicated that $P$. reticulata and $P$. wingei share the same male-heterogametic sex chromosome system (Nanda et al. 2014; Morris et al. 2018; Darolti et al. 2019). Analyses of coverage differences between males and females indicate that $\mathrm{Y}$ degeneration is restricted to the distal end of the sex chromosomes, in a region ancestral to P. reticulata and $P$. wingei

131 (designated as Stratum I) (Wright et al. 2017; Darolti et al. 2019; Darolti et al. 2020; Fraser et al. 2020), and suggest that $Y$ degeneration is slightly more exaggerated in $P$. wingei compared to $P$. reticulata. This nonrecombining region coincides with the previously mapped location of

134 the sex determining region in P. reticulata (Winge 1922; Winge 1927; Winge and Ditlevsen 1947; Traut and Winking 2001; Tripathi et al. 2009) and is also found across six natural guppy populations (Almeida et al. 2021).

We therefore assessed the signal of Fast- $X$ for genes in Stratum I (20-26Mb on P. reticulata chromosome $12 ; 17-20 \mathrm{Mb}$ on $P$. wingei syntenic chromosome to guppy sex chromosome). In P. reticulata, the rate of divergence for $\mathrm{X}$-linked genes in Stratum I was not significantly different than that for autosomal genes (Fig. 1A; supplementary Table S1). To exclude the possibility that we were lacking power in our analysis due to the small number of genes identified in Stratum I, we redid the analysis for $P$. reticulata using Ensembl coding sequences instead of our de novo generated transcripts. We were able to recover more than twice as many X-linked loci, however the estimates of divergence remain the same as those based on de novo transcripts (supplementary Fig. S2).

In P. wingei, genes in Stratum I show significantly higher rates of nonsynonymous and of synonymous substitutions (supplementary Table S1), however the overall rate of divergence is similar between the autosomes and Stratum I (Fig. 1B) and the Fast-X effect is not as 
149 accentuated as in $P$. picta (Fig. 2). Recent work has suggested that the $P$. wingei $\mathrm{X}$ and $\mathrm{Y}$ 150 chromosomes are somewhat more differentiated from each other compared to those of $P$. reticulata (Darolti et al. 2019), and there is also evidence for this from previous cytogenetic work (Nanda et al. 1992; Nanda et al. 2014). This greater divergence might explain the difference in $d_{\mathrm{N}} / d_{\mathrm{s}}$ estimates for X-linked genes in Stratum I that we observe between these two species, though the effect is negligible. However, note that following filtering (see methods), only a handful of $P$. wingei genes remained in Stratum I, and as such our statistical power to detect a significant Fast-X signal in this species is reduced.

If the guppy sex chromosomes represent a turnover event, we would expect the pattern of Fast-X in $P$. picta to still be observed throughout the $P$. reticulata and $P$. wingei sex chromosomes outside of Stratum I, as the majority of it accumulated prior to turnover. Thus, we next estimated rates of sequence divergence for the remainder of the $\mathrm{X}$ chromosome, excluding genes in Stratum I and genes on the PAR (see methods), as the high recombination events in the PAR can alter rates of evolution and polymorphism (Otto et al. 2011). The rate of mutation and mean $d_{N} / d_{s}$ on the $X$ chromosome were not different from that on the autosomes in either $P$. reticulata or $P$. wingei (Fig. 1A, B; Fig. 2; supplementary Table S1). This finding is consistent with the limited $\mathrm{Y}$ degeneration observed in these species (Wright et al. 2017; Darolti et al. 2019), and contradicts the Turnover Model. In addition, the Turnover Model also requires sex chromosome turnover events in the outgroup species to the $P$. reticulata-P. wingei clade (supplementary Fig. S2). We therefore estimated sequence divergence in the outgroups, $P$. latipinna and G. holbrooki, both species in which the guppy chromosome 12 has not been implicated as the sex chromosome. Our data fails to show elevated rates of evolution on the chromosome syntenic to the guppy $\mathrm{X}$ relative to the rest of the genome in either P. latipinna or G. holbrooki (supplementary Table S1). This result, together with our findings in $P$. reticulata and $P$. wingei argues against the Turnover Model.

Previous comparisons of replicate $P$. reticulata natural population have shown evidence for greater $\mathrm{X}-\mathrm{Y}$ divergence in replicate upstream, low predation populations relative to their downstream, high predation populations pair within Quare, Aripo and Yarra rivers (Wright et

177 al. 2017; Almeida et al. 2021). This pattern of divergence has occurred over a relatively short time span, at some point after the colonization of Trinidad at the last glacial maximum. 
coding sequence evolution in upstream compared to downstream populations. We find a significantly elevated $d_{N} / d_{S}$ on the $\mathrm{X}$ chromosome, excluding genes on the PAR and those on

182 Stratum I, compared to the autosomes in the Yarra upstream population only (supplementary Tables S2-4), and across all rivers we do not find a consistently stronger Fast-X effect in the upstream relative to the downstream populations (Fig. 3). Compared to the other rivers, the upstream population of the Yarra river shows the greatest increase in $Y$ divergence relative to the downstream population, measured by both male:female $F_{S T}$ and male-specific SNPS

187 (Almeida et al. 2021). X-Y divergence between upstream and downstream populations is 188 greater in Yarra compared to Quare and Aripo, and this might explain why a pattern of Fast-X effect in the upstream population is recovered in Yarra alone.

\section{Polymorphism and the underlying causes of Fast-X}

191 A higher $d_{N} / d_{s}$ on the $X$ chromosome relative to the rest of the genome could be the result of 192 both an increased genetic drift (Charlesworth et al. 1987; Mank et al. 2010) and an increased 193 efficacy of selection (Vicoso and Charlesworth 2009). Therefore, we used sequence and 194 polymorphism data together to test for the causes of elevated Fast-X evolution in P. picta. The 195 McDonald-Kreitman test contrasts the number of nonsynonymous and synonymous substitutions with polymorphisms, where an excess of nonsynonymous substitutions relative to polymorphisms is indicative of positive selection (McDonald and Kreitman 1991). Using this test, we detected no X-linked genes with signatures of positive selection in any of the Poeciliid species. However, the McDonald-Kreitman test is very conservative, as it is restricted to genes with sufficient numbers of substitutions and polymorphisms (Begun et al. 2007; Andolfatto 2008), and as such our analysis was limited to a few X-linked contigs.

To increase our statistical power to detect signatures of positive selection, we also used the direction of selection (DoS) test which is less sensitive to low cell counts (Stoletzki and EyreWalker 2011). For each contig, DoS calculates the difference between the proportion of nonsynonymous substitutions and the proportion of nonsynonymous polymorphisms, where a positive DoS indicates adaptive evolution (Stoletzki and Eyre-Walker 2011). Using this approach, we recovered more genes with signatures of positive selection, however the $X$ chromosome was not enriched in these genes compared to the rest of the genome in any of the species (supplementary Table S6). 
210 Next, we used polymorphism data alone to test for deviations from neutrality. A higher rate

211 of nonsynonymous to synonymous polymorphisms on the $\mathrm{X}$ relative to the autosomes is

212 indicative of reduced efficacy of selection to remove mildly deleterious mutations, while a

213 lower rate suggests purifying selection resulting from hemizygous exposure of deleterious

214 mutations in males. Our results show a lower, though not significantly, rate of nonsynonymous

215 polymorphisms in P. picta only (Table 1, supplementary Table S5), which may suggest that the

216 observed Fast-X effect in $P$. picta is influenced by purifying selection acting to remove

217 deleterious mutations on the X (Charlesworth et al. 1987; Vicoso and Charlesworth 2009).

\section{Discussion}

\section{The role of hemizygosity in Fast-X}

220 We observe a clear pattern of Fast-X in P. picta, as shown through the significantly higher rate of nonsynonymous substitutions for X-linked loci (supplementary Table S1) and significantly elevated rate of divergence on the $\mathrm{X}$ chromosome relative to the rest of the genome (Fig. $1 \mathrm{C}$, Fig. 2), consistent with the extensive Y chromosome degeneration in this species. In contrast, we did not observe significant patterns of Fast-X evolution in either $P$. reticulata or $P$. wingei. There is little evidence of loss of $Y$ gene coding sequence in either of these species (Darolti et al. 2019; Darolti et al. 2020), and very few, if any, genes are hemizygously expressed in males, even in the older Stratum I. Interestingly, the $P$. wingei $Y$ is somewhat more diverged than the Y in P. reticulata (Nanda et al. 1992; Nanda et al. 2014; Darolti et al. 2019), and we observe a slight, though non-significant Fast-X effect in Stratum I in the former. We also observe an effect in the Yarra upstream population, which shows the greatest $X-Y$ divergence across natural populations in Trinidad (Almeida et al. 2021). All this points to the key role of sex chromosome hemizygosity in driving Fast-X evolution.

233 Theory predicts that complete dosage compensation, whereby balance in expression between the sex chromosomes and the autosomes is restored, may facilitate a more pronounced FastX effect (Charlesworth et al. 1987; Mank et al. 2010). Under this theory, in systems with incomplete dosage compensation, where only a subset of the genes is compensated for but overall expression for $\mathrm{X}$-linked genes is reduced compared to the autosomes in males, $\mathrm{X}$-linked beneficial mutations may have lower expression and weaker phenotypic effects in males, 
evolved chromosome-wide dosage compensation, seemingly through a mechanism involving

241 the hyperexpression of the single $X$ in males (Darolti et al. 2019), and this may have contributed to the observed signature of Fast-X evolution.

There are two potential causes of Fast-X. Male heterogamety leads to hemizygous exposure of genes and therefore stronger purifying selection against recessive deleterious mutations and positive selection for recessive beneficial ones expressed in males (Charlesworth et al. 1987). Alternatively, the reduced effective population size of the $X$ relative to the autosomes diminishes the relative power of selection on the $X$, potentially leading to non-adaptive causes of Fast-X (Charlesworth et al. 1987) which do not necessarily require male hemizygosity, just recombination suppression between the $\mathrm{X}$ and $\mathrm{Y}$ chromosomes.

The role of hemizygosity and dosage compensation we observe in $P$. picta Fast-X suggest an adaptive mechanism, and our polymorphism analysis is consistent with greater purifying selection acting in males to remove recessive deleterious variation. However, although we were unable to differentiate adaptive and non-adaptive causes of Fast-X in our polymorphism data, polymorphism estimates are sensitive to demographic fluctuations (Tajima 1989; Pool and Nielsen 2007) and, thus, it is difficult to determine to what extent the Fast-X pattern in $P$. picta is adaptive using polymorphism data alone. Future work identifying true X-hemizygous loci in males and analyzing their patterns of sequence evolution may prove more revealing.

\section{Differentiating different models of sex chromosome origin}

Two models have been proposed for the origin of the sex chromosome system in this group.

260 The Parsimony Model posits that the sex chromosomes arose roughly 20 mya (supplementary Fig. S1) in the ancestor of the species that all share it and experienced different rates of $Y$ decay in different sub-clades (Darolti et al. 2019), possibly exacerbated by the evolution of complete dosage compensation in P. picta and close relatives (Metzger et al. 2021; Sandkam et al. 2021). The Turnover Model proposes that the sex chromosomes of $P$. reticulata represent a recent turnover event, and that the highly diverged $P$. picta and $P$. parae system arose well before the immediate ancestor of $P$. parae, $P$. picta, $P$. reticulata and $P$. wingei, and slowly diverged over time resulting in the heteromorphism observed (Charlesworth et al. 2021). Because the molecular signatures that accumulate on sex chromosomes remain 
able to critically test these alternative models. If the guppy sex chromosomes represent a turnover event, we would expect the pattern of Fast-X in P. picta to still be observed throughout the $P$. reticulata and $P$. wingei sex chromosomes outside of Stratum I, as the majority of it accumulated prior to turnover. In addition, the Turnover Model also requires sex chromosome turnover events in the outgroup species to the $P$. reticulata-P. wingei clade, and we would expect Fast-X in P. latipinna or G. holbrooki, where the ancient X chromosome is presumably now fully autosomal.

We do not observe signatures of Fast-X in $P$. reticulata and $P$. wingei that would be consistent with the Turnover Model, even after investigating multiple datasets (based on de novo transcripts of a lab population and replicate wild populations, and Ensembl coding sequences). Similarly, the lack of Fast-X in P. latipinna and G. holbrooki, the near outgroups to P. picta, $P$. reticulata, and $P$. wingei (Darolti et al. 2019), which would have also experienced sex chromosome turnover to alternative autosomes if the $P$. picta sex chromosomes are ancient, also argues against an ancient origin and turnover. Instead, our results support the Parsimony Model, and suggest that the sex chromosomes originated in the immediate ancestor of the $P$. picta-P. reticulata-P. wingei clade and divergence occurred more rapidly in $P$. picta, potentially accelerated by the evolution of complete $X$ chromosome dosage compensation in this species, which reduces selection to maintain $Y$ chromosome expression for dosage sensitive genes (Metzger et al. 2021).

\section{Materials and Methods}

\section{Sample collection and sequencing}

We have previously obtained tissue samples, extracted and sequenced RNA from the tails of three males and three females of P. reticulata, P. wingei, P. picta, P. latipinna and G. holbrooki (BioProject ID PRJNA353986, PRJNA528814) (Wright et al. 2017; Darolti et al. 2019). P. reticulata samples were obtained from our outbred laboratory population originating from the Quare River in Trinidad (Kotrschal et al. 2013). P. wingei samples were collected from our laboratory population established from a strain maintained by a UK fish fancier. P. picta samples were acquired from Guyana, while P. latipinna and G. holbrooki samples were obtained in Florida. All samples were collected in accordance with national and institutional ethical guidelines. 
300 We extracted RNA from each sample using the Qiagen RNeasy Kit, following the instructions

301 of the manufacturer. Library preparation and sequencing were performed at the University

302 of Oxford Wellcome Centre for Human Genetics, following standard Illumina protocols and 303 using the Illumina HiSeq 4000 platform. The data were quality assessed using FastQC v0.11.3

304 (www.bioinformatics.babraham.ac.uk/projects/fastqc) and trimmed with Trimmomatic v0.36 (Bolger 2014), removing adaptor sequences, reads with an average Phred score < 15 in a sliding window of four bases, reads with leading or trailing bases with a Phred score $<3$ and reads shorter than $50 \mathrm{bp}$ following trimming.

\section{Identifying orthogroups}

309 For each species, we mapped RNA-seq reads to a previously constructed species-specific 310 female de novo genome assembly (Wright et al. 2017; Darolti et al. 2019) using HISAT2 v2.0.4

311 (Kim et al. 2015), with the exception of $P$. latipinna for which a male genome assembly was 312 used, and a non-redundant set of transcripts in GTF file format was constructed using 313 StringTie v1.2.4 (Pertea et al. 2015). We filtered the transcripts for non-coding RNA (ncRNA) 314 by extracting transcript sequences with BEDtools getfasta (Quinlan and Hall 2010) and 315 removing transcripts with a BLAST hit to ncRNA sequences from Poecilia formosa 316 (PoeFor_5.1.2), Oryzias latipes (MEDAKA1), Gasterosteus aculeatus (BROADS1) and Danio 317 rerio (GRCz10) from Ensembl 104 (Flicek et al. 2014). Only genes with positional information on chromosomal fragments were kept for further analyses and the longest isoform for each target gene was selected. Lastly, we applied a minimum expression filter of 2 RPKM in at least half of the samples of each sex (minimum of two out of three individuals of either sex), resulting in 13,306 $P$. reticulata, 15,089 P. wingei, 13,156 P. picta, 14,468 P. latipinna and 21,861 G. holbrooki genic sequences.

323 We obtained coding sequences from the outgroup species $P$. formosa (PoeFor_5.1.2), Xiphophorus maculatus (Xipmac4.4.2) and O. latipes (MEDAKA1) from Ensembl 104 and extracted the longest isoform for each gene. Separately for each of our target species, we determined orthology across target and outgroup sequences using reciprocal BLASTn v2.7.1

327 (Altschul et al. 1990) with an e-value cut-off of $10 \mathrm{e}^{-10}$ and a minimum percentage identity of $30 \%$. For genes with multiple blast hits, we chose the top hit based on the highest BLAST score. Our analysis resulted in 7,296 $P$. reticulata, 7,253 P. wingei, 7,786 $P$. picta, 7,251 $P$. latipinna and 6,477 G. holbrooki orthogroups (four-way 1:1 orthologs). 


\section{Estimating sequence divergence across orthogroups}

332 For each contig of each orthogroup, we obtained open reading frames using 0 . latipes

333 (MEDAKA1) protein-coding sequences from Ensembl 104 and BLASTx v2.3.0 with an e-value

334 cut-off of $10 \mathrm{e}^{-10}$ and a minimum percentage identity of $30 \%$, excluding orthogroups without

335 BLASTx hits or valid protein-coding sequences. We aligned orthologous gene sequences with

336 PRANK v170427 (Löytynoja and Goldman 2008), using the rooted tree (((Target species, $P$.

337 formosa), X. maculatus), O. latipes), where the target species was in turn $P$. reticulata, $P$.

338 wingei, P. picta, P. latipinna and G. holbrooki. Alignments were then filtered to remove gaps.

339 To avoid false positive signals of adaptive evolution, poorly aligned or error-rich regions were

340 masked with SWAMP (Harrison et al. 2014). We ran SWAMP twice, first using a threshold of six nonsynonymous substitutions in a window size of 15 codons, and second using a threshold

342 of two and a window size of five. This approach eliminates sequencing errors that cause short

343 stretches of nonsynonymous substitutions as well as alignment errors that cause longer stretches of nonhomologous sequence due to variation in exon splicing or misannotation

345 (Harrison et al. 2014). To select these thresholds, we first applied a range of masking criteria on our datasets. We then ran the branch-site test for positive selection on the target species branches for both the unmasked and masked datasets. We visually inspected the alignments

348 of genes with the highest likelihood ratios and chose the masking criteria that was most efficient at reducing false positive rates. Finally, we discarded orthologs for which the alignment length was shorter than 300 bp following gap removal and masking, as these likely represent incomplete sequences.

To obtain divergence estimates for each orthogroup and calculate mean $d_{N} / d_{\mathrm{s}}$ across the target species branch, we used branch model (model=2, nssites=0) in the CODEML package in PAML v4.8 (Yang 2007), using the phylogeny ((Target species\#1, P. formosa), X. maculatus, O. latipes), where the target species was successively P. reticulata, P. wingei, P. picta, $P$. latipinna and $G$. holbrooki. To avoid inaccurate divergence estimates dues to mutational saturation and double hits, orthologous genes with $d_{s}>2$ were removed from subsequent analyses (Axelsson et al. 2008). 
361 previously identified pseudoautosomal regions $(0-5 \mathrm{Mb}$ and $>26 \mathrm{Mb}$ of $P$. reticulata

362 chromosome $12 ;>20 \mathrm{Mb}$ of P. wingei, P. picta, P. latipinna and G. holbrooki chromosomes

363 that are syntenic to the guppy chromosome 12 (Darolti et al. 2019)). In addition, it has recently been discovered that the $P$. reticulata reference genome, which served as reference for scaffold ordering and orientation for our $P$. reticulata de novo genome assembly, has a large inversion on the $\mathrm{X}$ chromosome that is specific to the guppy strain on which the reference genome assembly was built and is not present in any of our lab or wild-caught samples (Darolti et al. 2020; Almeida et al. 2021). We thus corrected for this inversion when excluding genes from the PAR and assigning genes to the sex-linked category in P. reticulata.

Separately for each genomic category, we extracted the number of nonsynonymous substitutions $\left(D_{\mathrm{N}}\right)$, the number of nonsynonymous sites $(\mathrm{N})$, the number of synonymous substitutions $\left(D_{S}\right)$ and the number of synonymous sites $(S)$. Taking into account alignment length, we calculated mean $d_{N}$ and mean $d_{\mathrm{S}}$ as the ratio of the number of substitutions across all orthologs in that group divided by the number of sites $\left(d_{N}=D_{N} / N ; d_{S}=D_{S} / S\right)$, thus avoiding bias from short sequences and the issue of infinitely high $d_{\mathrm{N}} / d_{\mathrm{s}}$ estimates due to very low $d_{\mathrm{s}}$ (Mank et al. 2007). We identified significant differences in $d_{N}, d_{\mathrm{s}}$ and $d_{\mathrm{N}} / d_{\mathrm{s}}$ between genomic categories using 1,000 permutation test replicates and we used bootstrapping with 1,000 replicates to determine $95 \%$ confidence intervals. The strength of the Faster-X effect was calculated for each target species as the ratio of the rate of divergence for the $\mathrm{X}$ chromosome over the rate of divergence for the autosomes $\mathrm{X}\left(d_{\mathrm{N}} / d_{\mathrm{S}}\right): \mathrm{A}\left(d_{\mathrm{N}} / d_{\mathrm{s}}\right)$.

\section{Additional sequence divergence analyses for $P$. reticulata}

382 For comparison to our estimates of rates of divergence based on de novo transcripts, we also estimated rates of sequence evolution in $P$. reticulata using publicly available coding sequences from Ensembl 104 (Guppy_female_1.0_MT). For this analysis, we followed the same steps outlined above in terms of extracting the longest isoform for each gene, identifying orthogroups, aligning target and outgroup sequences, masking and obtaining divergence estimates.

388 In addition, we have previously constructed high quality $P$. reticulata female de novo genome assemblies for replicate upstream and downstream populations of three rivers in Trinidad,

390 Quare, Aripo, and Yarra (Almeida et al. 2021). To each genome assembly, we mapped $P$. 
reticulata RNA-seq reads from our laboratory population and generated a non-redundant set

392 of transcripts for each wild population following the methods detailed above. For each upstream and downstream population, we then estimated rates of coding sequence evolution for the $X$ chromosome, excluding the PAR, and the autosomes by following the same pipeline described in the previous section.

\section{Polymorphism data}

397 To obtain polymorphism data, for each target species we first mapped female RNA-seq reads to the genome assembly using the STAR v2.4.2a aligner in two-pass mode (Dobin et al. 2013), and then called SNPs using SAMtools v1.3.1 mpileup (Li et al. 2009) with a minimum base quality of 20 and VarScan v2.3.9 mpileup2snp (Koboldt et al. 2012) with a minimum coverage of two, a minimum average quality of 20 , a minimum variant allele frequency of $1 \mathrm{e}^{-1}, p$ value of 0.05 and strand filter set to on. We next imposed that the polymorphism dataset also passes the filtering criteria used for calculating rates of sequence divergence. As such, we identified codons for which all sites pass the minimum coverage threshold of 20 , there are no alignment gaps following alignment with PRANK, and no ambiguity data (Ns) following masking with SWAMP. To make our dataset compatible for the McDonald-Kreitman test of selection, we only kept genes with both divergence and polymorphism data. Finally, for each genomic category, we calculated mean $p_{\mathrm{N}}$ and mean $p_{\mathrm{S}}$ as the ratio of the number of polymorphisms across all orthologs in that group divided by the number of sites $\left(p_{\mathrm{N}}=P_{\mathrm{N}} / \mathrm{N}\right.$; $\left.p_{\mathrm{S}}=P_{\mathrm{S}} / \mathrm{N}\right)$.

\section{Testing for selection using divergence and polymorphism data}

412 For each species, we estimated the number of genes evolving under neutral and adaptive

413 evolution using the McDonald-Kreitman test (McDonald and Kreitman 1991). Neutral theory 414 predicts that the ratio of nonsynonymous to synonymous changes within species $\left(p_{\mathrm{N}} / p_{\mathrm{S}}\right)$ 415 should be equal to that between species $\left(d_{N} / d_{s}\right)$ (McDonald and Kreitman 1991). As such, the 416 McDonald-Kreitman test identifies signatures of positive selection, where there is an excess 417 of nonsynonymous substitutions relative to polymorphisms $\left(d_{N} / d_{S}>p_{N} / p_{S}\right)$, and of relaxed 418 purifying selection, where there is a deficit of nonsynonymous substitutions compared to 419 polymorphisms $\left(d_{\mathrm{N}} / d_{\mathrm{S}}<p_{\mathrm{N}} / p_{\mathrm{S}}\right)$. To test for deviations from neutrality, for each contig, we used 420 a 2 x 2 contingency table and a Fisher's Exact Test in R v3.6.2 (R Core Team 2015). The power 421 of the McDonald-Kreitman test is limited with low table counts, therefore we restricted this 
analysis to genes for which the sum of each row and column in the contingency table was equal to or greater than six (Begun et al. 2007; Andolfatto 2008).

As the McDonald-Kreitman test is a very conservative test, we also used the divergence and polymorphism data to calculate the direction of selection statistic (DoS) (Stoletzki and EyreWalker 2011). For each gene, we calculated the difference between the proportion of nonsynonymous substitutions and polymorphisms (DoS $=D_{N}\left(D_{N}+D_{S}\right)-P_{N}\left(P_{N}+P_{S}\right)$ ), where positive DoS values are indicative of positive selection. We used Fisher's Exact test in $\mathrm{R}$ to test for significant differences in the proportion of genes under positive selection between the autosomes and the $\mathrm{X}$ chromosome in each species.

431 Lastly, we used the polymorphism data alone to test for an excess or deficit of nonsynonymous polymorphisms on the $\mathrm{X}$ chromosome compared to the autosomes, which would suggest a relaxed constraint or purifying selection, respectively. For this, we concatenated $P_{\mathrm{N}}$ and $P_{\mathrm{S}}$ estimates within each species and used Fisher's Exact test in $\mathrm{R}$ to test for significant differences in $P_{\mathrm{N}} / P_{\mathrm{S}}$ between the autosomes and the $\mathrm{X}$ chromosome.

\section{Acknowledgements}

437 This work was supported by the European Research Council (grant agreement 680951), a 438 Canada 150 Research Chair, and an NSERC Discovery Grant to J.E.M. We thank C. Lacy for the 439 fish illustrations and members of the Mank lab for helpful discussions and suggestions.

\section{$440 \quad$ Author Contributions}

441 J.E.M. and I.D. designed the research; I.D. and L.F. performed the research; I.D., L.F. and J.E.M. 442 analyzed the data; I.D., L.F. and J.E.M. wrote the manuscript.

\section{References}

444 Almeida P, Sandkam BA, Morris J, Darolti I, Breden F, Mank JE. 2021. Divergence and 445 remarkable diversity of the $Y$ chromosome in guppies. Mol Biol Evol. 38:619-633.

446 Altschul SF, Gish W, Miller W, Myers E, Lipman DJ. 1990. Basic local alignment search tool. J 447 Mol Biol. 215:403-410.

448 Andolfatto P. 2008. Controlling Type-I Error of the McDonald-Kreitman Test in Genomewide 449 Scans for Selection on Noncoding DNA. Genetics. 180:1767-1771.

450 Ávila V, De Procé SM, Campos JL, Borthwick H, Charlesworth B, Betancourt AJ. 2014. Faster$451 \quad X$ effects in two drosophila lineages. Genome Biol Evol. 6:2968-2982. 
Axelsson E, Hultin-Rosenberg L, Brandström M, Zwahlén M, Clayton DF, Ellegren H. 2008. Natural selection in avian protein-coding genes expressed in brain. Mol Ecol. 17:3008-3017.

Bachtrog D, Mank JE, Peichel CL, Kirkpatrick M, Otto SP, Ashman TL, Hahn MW, Kitano J, Mayrose I, Ming R, et al. 2014. Sex determination: why so many ways of doing it? PLoS Biol. 12:e1001899.

Bechsgaard J, Schou MF, Vanthournout B, Hendrickx F, Knudsen B, Settepani V, Schierup $\mathrm{MH}$, Bilde T. 2019. Evidence for faster $\mathrm{X}$ chromosome evolution in spiders. Mol Biol Evol. 36:1281-1293.

460 Begun DJ, Holloway AK, Stevens K, Hillier LW, Poh YP, Hahn MW, Nista PM, Jones CD, Kern $A D$, Dewey $C N$, et al. 2007. Population genomics: whole-genome analysis of polymorphism and divergence in Drosophila simulans. PLoS Biol. 5:2534-2559.

Bolger AMLMUB. 2014. Trimmomatic: a flexible trimmer for Illumina sequence data. Bioinformatics. 30:2114-2120. chromosomes and autosomes. Am Nat. 130:113-146. chromosome evolve? PLoS Genet. 17:e1009704. incomplete recombination suppression. Genome Biol Evol. 12:965-977.

Darolti I, Wright AE, Sandkam BA, Morris J, Bloch NI, Farré M, Fuller RC, Bourne GR, Larkin DM, Breden F, et al. 2019. Extreme heterogeneity in sex chromosome differentiation and dosage compensation in livebearers. Proc Natl Acad Sci USA. 116:19031-19036. TR. 2013. STAR: ultrafast universal RNA-seq aligner. Bioinformatics. 29:15-21.

478 Flicek P, Amode MR, Barrell D, Beal K, Billis K, Brent S, ..., Searle SMJ. 2014. Ensembl 2014. 479 Nucleic Acids Res. 42:749-755.

480 Fraser BA, Whiting JR, Paris JR, Weadick CJ, Parsons PJ, Charlesworth D, Bergero R, Bemm F, Hoffmann M, Kottler VA, et al. 2020. Improved reference genome uncovers novel sex-linked regions in the guppy (Poecilia reticulata). Genome Biol Evol. 12:1789-1805.

483 Harrison PW, Jordan GE, Montgomery SH. 2014. SWAMP : Sliding Window Alignment Masker for PAML. Evol Bioinform. 10:EBO-S18193.

485 Jaquiéry J, Peccoud J, Ouisse T, Legeai F, Prunier-Leterme N, Gouin A, Nouhaud P, Brisson 486 JA., Bickel R, Purandare S, et al. 2018. Disentangling the Causes for Faster-X Evolution in 487 Aphids. Genome Biol Evol. 10:507-520.

488 Kim D, Langmead B, Salzberg SL. 2015. HISAT: A fast spliced aligner with low memory 489 requirements. Nat Methods. 12:357-360.

490 Kirkpatrick M, Hall DW. 2004. Male-biased mutation, sex linkage, and the rate of adaptive 491 evolution. Evol. 58:437-440. 
Koboldt DC, Zhang Q, Larson DE, Shen D, McLellan MD, Lin L, Miller CA, Mardis ER, Ding L, Wilson RK. 2012. VarScan 2: Somatic mutation and copy number alteration discovery in cancer by exome sequencing. Genome Res. 22:568-576.

Kotrschal A, Rogell B, Bundsen A, Svensson B, Zajitschek S, Immler S, Maklakov AA, Kolm N. 2013. Experimental evidence for costs and benefits of evolving a larger brain. Curr Biol. 23:168-171.

Kousathanas A, Halligan DL, Keightley PD. 2014. Faster-X adaptive protein evolution in house mice. Genetics. 196:1131-1143.

500 Li H, Handsaker B, Wysoker A, Fennell T, Ruan J, Homer N, Marth G, Abecasis G, Durbin R. 2009. The sequence alignment / map format and SAMtools. Bioinformatics. 25:2078-2079.

502 Löytynoja A, Goldman N. 2008. Phylogeny-aware gap replacement prevents errors in 503 sequence alignment and evolutionary analysis. Science. 320:1632-1635.

504 Lu J, Wu C-I. 2005. Weak selection revealed by the whole-genome comparison of the $X$ 505 chromosome and autosomes of human and chimpanzee. PNAS. 102:4063-4067.

506 Mank JE, Axelsson E, Ellegren H. 2007. Fast-X on the Z: rapid evolution of sex-linked genes in 507 birds. Genome Res. 17:618-624.

508 Mank JE, Vicoso B, Berlin S, Charlesworth B. 2010. Effective population size and the Faster-X effect: empirical results and their interpretation. Evolution. 64:663-674.

510 McDonald JH, Kreitman M. 1991. Adaptive protein evolution at the ADH locus in Drosophila. 511 Nature. 351:652-654.

512 Meisel RP, Connallon T. 2013. The faster-X effect: integrating theory and data. Trends 513 Genet. 29:537-544.

514 Metzger DCH, Sandkam BA, Darolti I, Mank JE. 2021. Rapid evolution of complete dosage 515 compensation in Poecilia. Genome Biol Evol. 13:evab155.

516 Morris J, Darolti I, Bloch NI, Wright AE, Mank JE. 2018. Shared and Species-Specific Patterns 517 of Nascent Y Chromosome Evolution in Two Guppy Species. Genes. 9:238.

518 Nanda I, Schartl M, Feichtinger W, Epplen JT, Schmid M. 1992. Early stags of sex 519 chromosome differentiation in fish as analysed by simple repetitive DNA sequences. 520 Chromosoma. 101:301-310.

521 Nanda I, Schories S, Tripathi N, Dreyer C, Haaf T, Schmid M, Schartl M. 2014. Sex chromosome polymorphism in guppies. Chromosoma. 123:373-383.

523 Otto SP, Pannell JR, Peichel CL, Ashman T-L, Charlesworth D, Chippindale AK, Delph LF, 524 Guerrero RF, Scarpino S V, McAllister BF. 2011. About PAR: the distinct evolutionary 525 dynamics of the pseudoautosomal region. Trends Genet. 27:358-367.

526 Pertea M, Pertea GM, Antonescu CM, Chang TC, Mendell JT, Salzberg SL. 2015. StringTie 527 enables improved reconstruction of a transcriptome from RNA-seq reads. Nat Biotechnol. 528 33:290-295.

529 Pinharanda A, Rousselle M, Martin SH, Hanly JJ, Davey JW, Kumar S, Galtier N, Jiggins CD. 530 2019. Sexually dimorphic gene expression and transcriptome evolution provide mixed 531 evidence for a fast-Z effect in Heliconius. J Evol Biol. 32:194-204. 
Pool JE, Nielsen R. 2007. Population size changes reshape genomic patterns of diversity. J Org Evol. 61:30001-3006.

Quinlan AR, Hall IM. 2010. Genome analysis BEDTools: A flexible suite of utilities for comparing genomic features. Bioinformatics. 26:841-842.

R Core Team. 2015. R: A language and environment for statistical computing. Vienna, Austria: R Foundation for Statistical Computing. Available from: https//www.r-project.org. Accessed August 25, 2021.

Rousselle M, Faivre N, Ballenghien M, Galtier N, Nabholz B. 2016. Hemizygosity enhances purifying selection: Lack of fast-Z evolution in two satyrine butterflies. Genome Biol Evol. 8:3108-3119.

Sackton TB, Corbett-Detig RB, Nagaraju J, Vaishna L, Arunkumar KP, Hartl DL. 2014. Positive selection drives faster-Z evolution in silkmoths. Evolution. 68:2331-2342.

Sandkam BA, Almeida P, Darolti I, Furman BLS, van der Bijl W, Morris J, Bourne GR, Breden F, Mank JE. 2021. Extreme $Y$ chromosome polymorphism corresponds to five male reproductive morphs of a freshwater fish. Nat Ecol Evol. 5:939-948.

Stevenson BJ, Iseli C, Panji S, Zahn-Zabal M, Hide W, Old LJ, Simpson AJ, Jongeneel CV. 2007. Rapid evolution of cancer/testis genes on the X chromosome. BMC Genomics. 8:1-11.

Stoletzki N, Eyre-Walker A. 2011. Estimation of the neutrality index. Mol Biol Evol. 28:63-70.

Tajima F. 1989. The effect of change in population size on DNA polymorphism. Genetics. 123:597-601.

Traut W, Winking H. 2001. Meiotic chromosomes and stages of sex chromosome evolution in fish: zebrafish, platyfish and guppy. Chromosom Res. 9:659-672.

Tripathi N, Hoffmann M, Willing EM, Lanz C, Weigel D, Dreyer C. 2009. Genetic linkage map of the guppy, Poecilia reticulata, and quantitative trait loci analysis of male size and colour variation. Proc Biol Sci. 276:2195-2208.

Vicoso B, Bachtrog D. 2013. Reversal of an ancient sex chromosome to an autosome in Drosophila. Nature. 499:332-335.

Vicoso B, Charlesworth B. 2006. Evolution on the X chromosome: unusual patterns and processes. Nat Rev Genet. 7:645.

Vicoso B, Charlesworth B. 2009. The deficit of male-biased genes on the D. melanogaster X chromosome is expression-dependent: A consequence of dosage compensation? J Mol Evol. 68:576-583.

Winge Ö. 1922. One-sided masculine and sex-linked inheritance in Lebistes reticulatus. J Genet. 12:145-162.

Winge Ö. 1927. The location of eighteen genes in Lebistes reticulatus. J Genet. 18:1-43.

Winge Ö, Ditlevsen E. 1947. Colour inheritance and sex determination in Lebistes. Heredity. 1:65-83.

Wright AE, Darolti I, Bloch NI, Oostra V, Sandkam B, Buechel SD, Kolm N, Breden F, Vicoso B, Mank JE. 2017. Convergent recombination suppression suggests role of sexual selection in 
bioRxiv preprint doi: https://doi.org/10.1101/2021.09.03.458929; this version posted September $5,2021$. The copyright holder for this preprint (which was not certified by peer review) is the author/funder, who has granted bioRxiv a license to display the preprint in perpetuity. It is made available under aCC-BY-NC-ND 4.0 International license.

571 guppy sex chromosome formation. Nat Commun. 8:14251.

572 Wright AE, Harrison PW, Zimmer F, Montgomery SH, Pointer MA, Mank JE. 2015. Variation

573 in promiscuity and sexual selection drives avian rate of Faster-Z evolution. Mol Ecol.

$574 \quad 24: 1218-1235$.

575 Wright AE, Mank JE. 2013. The scope and strength of sex-specific selection in genome 576 evolution. J Evol Biol. 26:1841-1853.

577 Yang Z. 2007. PAML 4: phylogenetic analysis by maximum likelihood. Mol Biol Evol. $578 \quad 24: 1586-1591$.

579 


\section{$580 \quad$ Figure Legends}

581 Figure 1. Estimates of the rate of divergence $\left(d_{N} / d_{s}\right)$ for autosomal and X-linked genes in (A) $582 \quad$ P. reticulata, (B) P. wingei, (C) P. picta, (D) P. latipinna and (E) G. holbrooki. For all species, 583 the $X$ chromosome category excludes genes on the PAR. Additionally, in P. reticulata and $P$. 584 wingei, the $X$ chromosome category excludes genes in Stratum I. In P. latipinna and $G$. 585 holbrooki, the $X$ chromosome represents the chromosome syntenic to guppy chromosome 586 12. 95\% confidence intervals are based on bootstrapping with 1,000 replicates. Differences 587 between autosomal and X-linked loci are based on 1,000 replicates permutation tests. Only 588 significant differences are shown $(p$ value $<0.01)$.

589 Figure 2. Fast-X effect, calculated as the ratio of $d_{\mathrm{N}} / d_{\mathrm{S}}$ for the $\mathrm{X}$ chromosome (excluding 590 the PAR) to that of the autosomes, across the poeciliids. In P. latipinna and G. holbrooki, 591 the $X$ chromosome represents the chromosome syntenic to guppy X. 95\% confidence 592 intervals are based on bootstrapping with 1,000 replicates. Green color shading represents 593 the extent of sex chromosome degeneration (mild degeneration in light green to extreme 594 degeneration in dark green), while species in which the guppy chromosome 12 has not been 595 implicated as the sex chromosome are shown in grey.

596 Figure 3. Estimates of rates of evolution in wild $\boldsymbol{P}$. reticulata populations. (A) Fast-X effect across $P$. reticulata upstream, low predation populations (orange) and downstream, high 598 predation populations (black) in the Quare, Aripo and Yarra rivers. The Fast- $X$ effect is 599 calculated as the ratio of the rate of nonsynonymous over synonymous substitutions for the $600 \mathrm{X}$ chromosome, excluding the PAR, relative to that for the autosomes. 


\section{Tables}

603

Table 1. Differences between nonsynonymous and synonymous polymorphisms on the autosomes and $X$ chromosome of each species. For all species, the $X$ chromosome category excludes genes on the PAR.

\begin{tabular}{|c|c|c|c|c|}
\hline Species & Category & $P_{\mathrm{N}}$ & $P_{s}$ & $p$ value $^{a}$ \\
\hline \multirow{2}{*}{ P. reticulata } & Autosomes & 1,890 & 5,566 & \multirow{2}{*}{$p=0.76$} \\
\hline & X chromosome & 59 & 184 & \\
\hline \multirow{2}{*}{ P. wingei } & Autosomes & 650 & 1,783 & \multirow{2}{*}{$p=0.69$} \\
\hline & X chromosome & 18 & 56 & \\
\hline \multirow{2}{*}{ P. picta } & Autosomes & 837 & 2,178 & \multirow{2}{*}{$p=0.24$} \\
\hline & X chromosome & 12 & 47 & \\
\hline \multirow[b]{2}{*}{ P. latipinna } & Autosomes & 1,165 & 3,265 & \multirow[b]{2}{*}{$p=0.24$} \\
\hline & $\begin{array}{c}\text { Chromosome } \\
\text { syntenic to guppy } X\end{array}$ & 52 & 107 & \\
\hline \multirow[b]{2}{*}{ G. holbrooki } & Autosomes & 1,212 & 4270 & \multirow[b]{2}{*}{$p=0.08$} \\
\hline & $\begin{array}{c}\text { Chromosome } \\
\text { syntenic to guppy X }\end{array}$ & 28 & 144 & \\
\hline
\end{tabular}


bioRxiv preprint doi: https://doi.org/10.1101/2021.09.03.458929; this version posted September 5, 2021. The copyright holder for this

preprint (which was not certified by peer review) is the author/funder, who has granted bioRxiv a license to display the preprint in perpetuity. It is made available under aCC-BY-NC-ND 4.0 International license.

\section{Figures}

$606 \quad$ Fig. 1
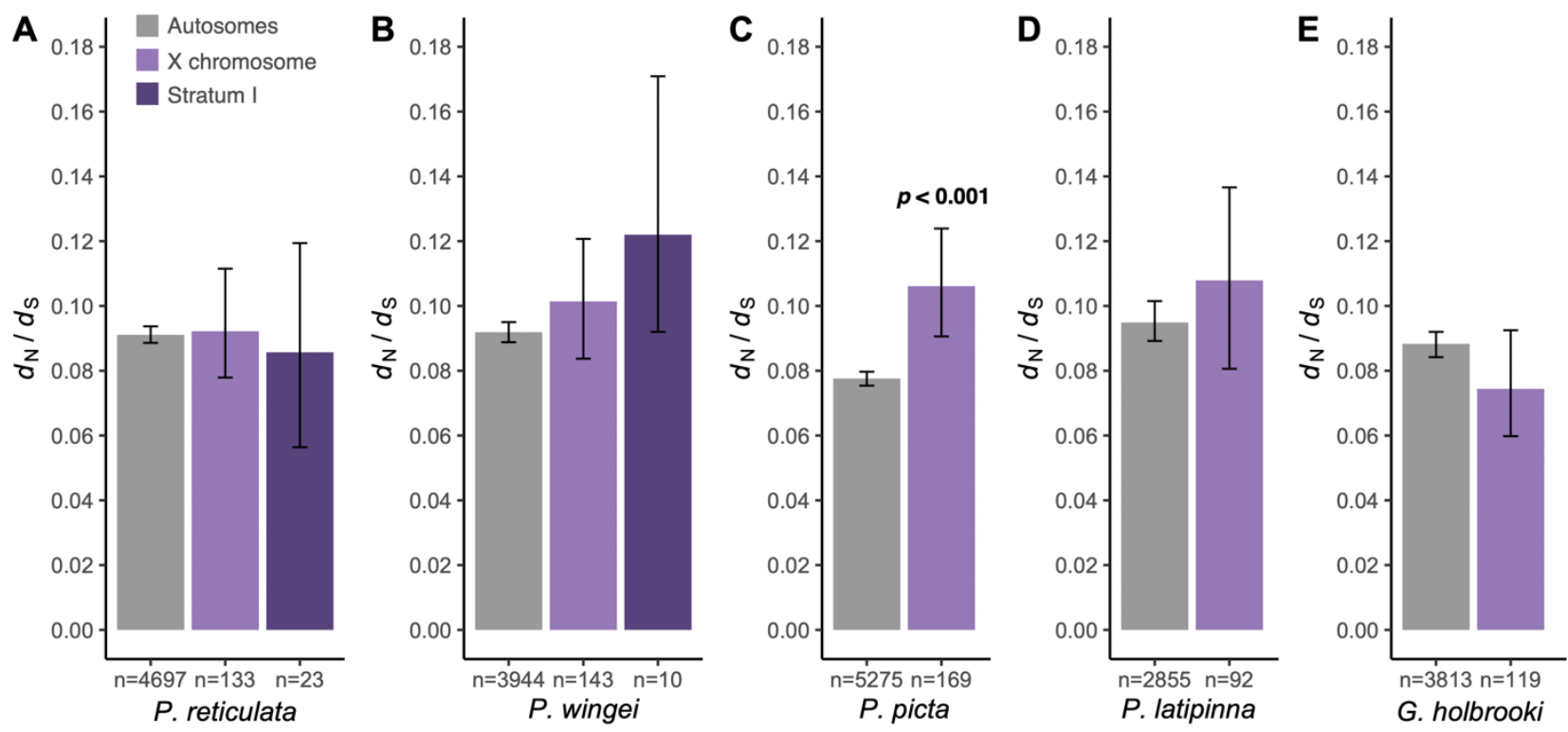
bioRxiv preprint doi: https://doi.org/10.1101/2021.09.03.458929; this version posted September 5, 2021. The copyright holder for this preprint (which was not certified by peer review) is the author/funder, who has granted bioRxiv a license to display the preprint in perpetuity. It is made available under aCC-BY-NC-ND 4.0 International license.

608

Fig. 2




bioRxiv preprint doi: https://doi.org/10.1101/2021.09.03.458929; this version posted September 5, 2021. The copyright holder for this preprint (which was not certified by peer review) is the author/funder, who has granted bioRxiv a license to display the preprint in perpetuity. It is made available under aCC-BY-NC-ND 4.0 International license.

$610 \quad$ Fig. 3



